\title{
A MEAN-VALUE THEOREM FOR SOME EIGENFUNCTIONS OF THE LAPLACE-BELTRAMI OPERATOR ON THE UPPER-HALF SPACE
}

\author{
Sirkka-Liisa Eriksson and Heikki Orelma \\ Tampere University of Technology, Department of Mathematics \\ P.O. Box 553, 33101 Tampere, Finland; sirkka-liisa.eriksson@tut.fi \\ Tampere University of Technology, Department of Mathematics \\ P.O. Box 553, 33101 Tampere, Finland; heikki.orelma@tut.fi
}

\begin{abstract}
In this paper we study a mean-value property for solutions of the eigenvalue equation of the Laplace-Beltrami operator

$$
\Delta_{l b} h=-(n-1) h
$$

with respect to the volume and the surface integrals on the Poincare upper-half space $\mathbf{R}_{+}^{n+1}=$ $\left\{\left(x_{0}, \ldots, x_{n}\right) \in \mathbf{R}^{n+1}: x_{n}>0\right\}$ with the Riemannian metric $d s^{2}=\frac{d x_{0}^{2}+d x_{1}^{2}+\cdots+d x_{n}^{2}}{x_{n}^{2}}$.
\end{abstract}

\section{Preliminaries}

In this section we recall the Laplace-Beltrami operator in the Poincaré upper-half space and formulate its connections with the so called hypermonogenic functions. Let us denote $\mathbf{R}_{+}^{n+1}=\left\{\left(x_{0}, x_{1}, \ldots, x_{n}\right) \in \mathbf{R}^{n+1}: x_{n}>0\right\}$. The Poincare half-space is the Riemannian manifold $\left(\mathbf{R}_{+}^{n+1}, d s^{2}\right)$, where the Riemannian metric is

$$
d s^{2}=\frac{d x_{0}^{2}+d x_{1}^{2}+\cdots+d x_{n}^{2}}{x_{n}^{2}} .
$$

The Laplace-Beltrami operator on the Poincare upper-half space is the operator (details are available for example in [7])

$$
\Delta_{l b} f=x_{n}^{2} \Delta f-(n-1) x_{n} \frac{\partial f}{\partial x_{n}},
$$

where $f: \Omega \rightarrow \mathbf{R}$ is a smooth enough function defined on an open subset $\Omega$ of $\mathbf{R}_{+}^{n+1}$ and $\Delta=\frac{\partial^{2}}{\partial x_{0}^{2}}+\cdots+\frac{\partial^{2}}{\partial x_{n}^{2}}$. The solutions of the Laplace-Beltrami equation $\Delta_{l b} f=0$ are called hyperbolic harmonic functions.

The Clifford algebra $\mathscr{C} \ell_{0, n}$ is the free associative algebra with unit generated by the symbols $e_{1}, \ldots, e_{n}$ together with the defining relations

$$
e_{i} e_{j}+e_{j} e_{i}=-2 \delta_{i j}
$$

for $i, j=1, \ldots, n$. As a vector space the dimension of the Clifford algebra $\mathscr{C} \ell_{0, n}$ is $2^{n}$. A canonical basis is given by $e_{A}=e_{a_{1}} \cdots e_{a_{k}}$, where $A=\left\{a_{1}, \ldots, a_{k}\right\} \subset\{1, \ldots, n\}$ and $1 \leq a_{1}<\ldots<a_{k} \leq n$. In particular, we denote $e_{\emptyset}=e_{0}=1$ and $e_{\{j\}}=e_{j}$. The

doi:10.5186/aasfm.2011.3606

2010 Mathematics Subject Classification: Primary 30A05; Secondary 30A05, 30 F45.

Key words: Laplace-Beltrami operator, mean-value theorem, hypermonogenic function, hyperbolic harmonic function. 
$(n+1)$-dimensional Euclidean space $\mathbf{R}^{n+1}$ is a subspace of $\mathscr{C} \ell_{0, n}$ under the canonical embedding

$$
\left(x_{0}, x_{1}, \ldots, x_{n}\right) \mapsto \sum_{j=0}^{n} x_{j} e_{j}
$$

and thus we may assume that $\mathbf{R}^{n+1} \subset \mathscr{C} \ell_{0, n}$. An element $a \in \mathscr{C} \ell_{0, n}$ is called a Clifford number and often the algebra $\mathscr{C} \ell_{0, n}$ is called the algebra of Clifford numbers. Elements $\mathbf{x}=\sum_{j=1}^{n} x_{j} e_{j} \in \mathscr{C} \ell_{0, n}$ are called vectors. Thus we see that an element $x$ of $\mathbf{R}^{n+1}$ may be written as

$$
x=x_{0}+\mathbf{x}
$$

with $\mathbf{x}=x_{1} e_{1}+\cdots+x_{n} e_{n}$ and it is called a paravector.

The conjugation is the algebra anti-automorphism on the Clifford algebra defined by $\bar{x}=x_{0}-\mathbf{x}$, that is, if $a, b \in \mathscr{C} \ell_{0, n}$, then $\overline{a b}=\bar{b} \bar{a}$. Also, $\mathbf{x}^{2}=\mathbf{x} \mathbf{x}=-x_{1}^{2}-\cdots-x_{n}^{2}$. Thus we may compute

$$
x \bar{x}=\left(x_{0}+\mathbf{x}\right)\left(x_{0}-\mathbf{x}\right)=x_{0}^{2}+x_{1}^{2}+\cdots+x_{n}^{2}
$$

for $x \in \mathbf{R}^{n+1}$. The Euclidean norm is then $|x|^{2}=x \bar{x}=\bar{x} x$. The main-involution is the algebra automorphism denoted and defined by $x^{\prime}=x_{0}-\mathbf{x}$, that is, if $a, b \in \mathscr{C} \ell_{0, n}$, then $(a b)^{\prime}=a^{\prime} b^{\prime}$.

Let us consider the Clifford algebra valued functions $f: \Omega \rightarrow \mathscr{C} \ell_{0, n}$, where $\Omega \subset \mathbf{R}_{+}^{n+1}$ is an open subset. Since the Clifford algebra $\mathscr{C} \ell_{0, n}$ is generated by the symbols $e_{1}, \ldots, e_{n}$, we obtain that then the Clifford algebra $\mathscr{C} \ell_{0, n-1}$ is generated by the symbols $e_{1}, \ldots, e_{n-1}$. Hence each $a \in \mathscr{C} \ell_{0, n}$ may be represented in the form

$$
a=b+c e_{n},
$$

where $b, c \in \mathscr{C} \ell_{0, n-1}$. We abbreviate $P a=b$ and $Q a=c$ and $Q^{\prime} a=(Q a)^{\prime}$ and $P^{\prime} a=(P a)^{\prime}$. Then we define the modified Dirac operator by

$$
M f=D f+\frac{n-1}{x_{n}} Q^{\prime} f,
$$

where $D=\frac{\partial}{\partial x_{0}}+e_{1} \frac{\partial}{\partial x_{1}}+\cdots+e_{n} \frac{\partial}{\partial x_{n}}$ is the Dirac operator on $\mathbf{R}^{n+1}$. The theory of null-solutions of the modified Dirac operator is called hyperbolic function theory, see, e.g., [4].

The function $f: \Omega \rightarrow \mathscr{C} \ell_{0, n}$ is called a hypermonogenic on $\Omega$ if $M f(x)=0$ for each $x \in \Omega$. Hypermonogenic functions have many nice function theoretic properties, for example, they have Cauchy-type integral formulas. Also, the function $x \mapsto x^{k}$, where $k \in \mathbf{Z}$, is hypermonogenic. Many properties and more references can be found from the survey article [4].

The conjugate of the modified Dirac operator is defined by

$$
\bar{M} f=\bar{D} f-\frac{n-1}{x_{n}} Q^{\prime} f,
$$

where $\bar{D}=\frac{\partial}{\partial x_{0}}-e_{1} \frac{\partial}{\partial x_{1}}-\cdots-e_{n} \frac{\partial}{\partial x_{n}}$.

In the hyperbolic function theory we define hyperbolic harmonic functions $f: \Omega \rightarrow$ $\mathscr{C} \ell_{0, n}$ as solutions of the equation

$$
\bar{M} M f(x)=0
$$

for $x \in \Omega$. 
The next theorem give us a connection between hypermonogenic functions and hyperbolic harmonic functions. Also, we see that the equation in above is really a good generalization for real-valued hyperbolic harmonic functions.

Theorem 1.1. [2] Let $\Omega \subset \mathbf{R}_{+}^{n+1}$ be an open subset and let $f: \Omega \rightarrow \mathscr{C} \ell_{0, n}$ be a twice differentiable function. Then

$$
P(\bar{M} M f)=\Delta P f-\frac{n-1}{x_{n}} \frac{\partial P f}{\partial x_{n}}
$$

and

$$
Q(\bar{M} M f)=\Delta Q f-\frac{n-1}{x_{n}} \frac{\partial Q f}{\partial x_{n}}+(n-1) \frac{Q f}{x_{n}^{2}} .
$$

If $f$ is hypermonogenic, then $P f$ satisfies the equation

$$
\Delta P f-\frac{n-1}{x_{n}} \frac{\partial P f}{\partial x_{n}}=0
$$

and $Q f$ satisfies the equation

$$
\Delta Q f-\frac{n-1}{x_{n}} \frac{\partial Q f}{\partial x_{n}}+(n-1) \frac{Q f}{x_{n}^{2}}=0 .
$$

Thus we see that the $Q$-part of a hypermonogenic function is a solution of the following eigenvalue equation

$$
\Delta_{l b} h=-(n-1) h .
$$

In the next section we shall study more detailed what is the structure of the above eigenfunctions.

Also, we see that the $P$-part of a hypermonogenic function is a direct generalization of a real-valued hyperbolic harmonic function. For a $\mathscr{C} \ell_{0, n-1}$-valued function, especially for the $P$-part of a hypermonogenic function, we obtained the following structure theorem.

Theorem 1.2. [5] Let $\Omega \subset \mathbf{R}_{+}^{n+1}$ be open and $g: \Omega \rightarrow \mathscr{C} \ell_{0, n-1}$ be a differentiable function. The following properties are equivalent.

(a) $g$ is a solution of the equation

$$
\Delta g-\frac{n-1}{x_{n}} \frac{\partial g}{\partial x_{n}}=0
$$

(b) $g$ is smooth and

$$
g(a)=\frac{1}{\omega_{n+1} \sinh ^{n} R_{h}} \int_{\partial B_{h}\left(a, R_{h}\right)} g(x) d \sigma_{h}(x)
$$

for all $\overline{B_{h}\left(a, R_{h}\right)} \subset \Omega$. In the formula $\omega_{n+1}$ denotes the surface area of the $n$-dimensional unit sphere.

(c) $g$ is smooth and

$$
g(a)=\frac{1}{V\left(B_{h}\left(a, R_{h}\right)\right)} \int_{B_{h}\left(a, R_{h}\right)} g(x) d x_{h}(x)
$$

for all $\overline{B_{h}\left(a, R_{h}\right)} \subset \Omega$, where $V\left(B_{h}\left(a, R_{h}\right)\right)=\sigma_{n} \int_{0}^{R_{h}} \sinh ^{n} t d t$ is the volume of the ball $B_{h}\left(a, R_{h}\right)$. 
In the previous theorem $B_{h}\left(a, R_{h}\right)$ is the hyperbolic ball with the center $a$ and the radius $R_{h}$. In the next section we shall give more detailed description for it. Since $\mathbf{R}$ is a canonical subset of $\mathscr{C} \ell_{0, n}$, we obtain the following obvious corollary.

Corollary 1.3. The preceding theorem is true also for real valued functions.

In the next section we shall state and prove a similar theorem for the preceding eigenfunctions.

\section{A mean-value theorem for some eigenfunctions of the Laplace-Beltrami operator}

Our aim is to give a detailed proof for the following structure theorem of the eigenfunctions represented in the previous section. First we recall a few basic facts from the hyperbolic geometry. A more detailed survey to the topic is available in [6]. In [6] it is shown that the hyperbolic ball with the radius $R_{h}$ and the center $a$ is the Euclidean ball with the center $\tau\left(a, R_{h}\right)$ and the radius $R_{e}\left(a, R_{h}\right)$,

$$
B_{h}\left(a, R_{n}\right)=\left\{x \in \mathbf{R}_{+}^{n+1}:\left|x-\tau\left(a, R_{h}\right)\right|<R_{e}\left(a, R_{h}\right)\right\},
$$

where

$$
\tau\left(a, R_{h}\right)=a_{0}+a_{1} e_{1}+\cdots+a_{n-1} e_{n-1}+a_{n} e_{n} \cosh R_{h}
$$

and

The $n$-form

$$
R_{e}\left(a, R_{h}\right)=a_{n} \sinh R_{h}
$$

$$
d \sigma=\sum_{j=0}^{n}(-1)^{j} e_{j} d \widehat{x}_{j}
$$

is often very useful vector valued differential form on $\mathbf{R}_{+}^{n+1}$, where $d \widehat{x}_{j}=d x_{0} \cdots d x_{j-1}$. $d x_{j+1} \cdots d x_{n}$.

Let $K$ be an $(n+1)$-dimensional manifold-with-boundary. On the boundary $\partial K$ the form $d \sigma$ admits the representation $d \sigma=\nu d S$, where $\nu$ is the outer unit normal vector field and $d S$ a scalar $n$-form. The corresponding surface form on the hyperbolic space is $d \sigma_{h}=\frac{d \sigma}{x_{n}^{n}}$ and if $d x$ is the volume form on the Euclidean space then the corresponding hyperbolic form is $d x_{h}=\frac{d x}{x_{n}^{n+1}}$. More detailed introduction to integration and certain differential forms in the Poincaré upper-half space can be found from $[6]$.

Theorem 2.1. Let $\Omega \subset \mathbf{R}_{+}^{n+1}$ be an open subset and let $h: \Omega \rightarrow \mathscr{C} \ell_{0, n-1}$ be a smooth function. The following properties are equivalent:

(i) $h$ is an eigenfunction of the Laplace-Beltrami operator with the eigenvalue $-(n-1)$, i.e, is a solution of

$$
\Delta_{l b} h(x)=-(n-1) h(x)
$$

for $x \in \Omega$.

(ii)

where

$$
h(a)=\frac{1}{\omega_{n+1} \psi\left(R_{h}\right)} \int_{\partial B_{h}\left(a, R_{h}\right)} h(x) d \sigma_{h}(x),
$$

$$
\psi\left(R_{h}\right)=\sinh R_{h} \int_{0}^{R_{h}} \sinh ^{n-2}(t) d t
$$


whenever $\overline{B\left(a, R_{h}\right)} \subset \Omega$.

(iii)

$$
h(a)=\frac{n-1}{\omega_{n+1} \phi\left(R_{h}\right)} \int_{B_{h}\left(a, R_{h}\right)} h(x) d x_{h},
$$

where $\omega_{n+1}$ is the surface area of the $(n+1)$-unit sphere and

$$
\phi\left(R_{h}\right)=(n-1) \cosh R_{h} \int_{0}^{R_{h}} \sinh ^{n-2}(t) d t-\sinh ^{n-1} R_{h}
$$

whenever $\overline{B\left(a, R_{h}\right)} \subset \Omega$.

The corresponding result in the case $n=2$ is already known. Leutwiler proved the theorem in his paper [8] using Green's functions which are simple in the case $n=2$. Authors wishes to emphasize that the methods of Leutwiler are available only in the special case $n=2$ since the Green's functions have much more complicated form in higher dimensions.

The first consequence is the following remark.

Corollary 2.2. The preceding theorem is true also for functions $h: \Omega \rightarrow \mathbf{R}$.

The proof of the theorem is based on a sequence of lemmata. First we recall the Cauchy's formula for the $Q$-part of a hypermonogenic function and other useful results.

Proposition 2.3. [1] If $f$ is a hypermonogenic function on $\Omega$ and $K \subset \Omega$ is an oriented $(n+1)$-dimensional manifold-with-boundary, then for each $a \in K$ we have

$$
Q f(a)=\frac{2^{n} a_{n}^{n-1}}{\omega_{n+1}} \int_{\partial K} Q(q(x, a) \nu(x) f(x)) d S(x),
$$

where $d S$ is the scalar surface element, $\nu$ is the outer unit normal vector field, and

$$
q(x, a)=-\frac{1}{2(n-1)} \bar{D} \frac{1}{|x-a|^{n-1}|x-\widehat{a}|^{n-1}}=\frac{1}{2} \frac{(x-a)^{-1}+(x-\widehat{a})^{-1}}{|x-a|^{n-1}|x-\widehat{a}|^{n-1}} .
$$

The kernel in the above integral admits the following expression.

Theorem 2.4. [6]

$$
q(x, a)=\frac{\overline{(x-\tau(a, x))} \cosh d_{h}(x, a)-a_{n} \sinh ^{2} d_{h}(x, a) e_{n}}{\left(2 a_{n} x_{n}\right)^{n} \sinh ^{n+1} d_{h}(x, a)},
$$

where

$$
\tau(a, x)=a_{0}+a_{1} e_{1}+\cdots+a_{n-1} e_{n-1}+a_{n} \cosh d_{h}(x, a) e_{n}
$$

and $d_{h}$ is the distance function with respect to the hyperbolic metric.

Also we need the following integration result. We define a generalized version of the modified Dirac operator by

$$
M_{n} f=D f-\frac{n}{x_{n}} Q^{\prime} f .
$$

Theorem 2.5. [3] Let $\Omega$ be an open subset of $\mathbf{R}_{+}^{n+1}$. If $K \subset \Omega$ is an oriented $(n+1)$-dimensional manifold-with-boundary and $g$ is a smooth Clifford algebra-valued function on $\Omega$, then

$$
\int_{\partial K} P(\nu(x) g(x)) \frac{d S(x)}{x_{n}^{n}}=\int_{K} P\left(M_{n} g(x)\right) \frac{d x}{x_{n}^{n}} .
$$


Using the preceding resukt we are able to prove the following lemma.

Lemma 2.6. Assume that $f$ is hypermonogenic on $\Omega$ and $\overline{B_{h}\left(a, R_{h}\right)} \subset \Omega$. Then

$$
\int_{\partial B_{h}\left(a, R_{h}\right)} Q\left(\frac{e_{n} \nu(x) f(x)}{x_{n}^{n}}\right) d S(x)=\int_{B_{h}\left(a, R_{h}\right)} Q f(x) d x_{h} .
$$

Proof. It is easy to see that $Q\left(e_{n} \nu(x) f(x)\right)=P^{\prime}(\nu(x) f(x))$. Using Theorem 2.5 we have

Since

$$
\int_{\partial B_{h}\left(a, R_{h}\right)} Q\left(\frac{e_{n} \nu(x) f(x)}{x_{n}^{n}}\right) d S(x)=\int_{B_{h}\left(a, R_{h}\right)} P^{\prime}\left(M_{n} f(x)\right) \frac{d x}{x_{n}^{n}} .
$$

we obtain

$$
M f(x)=D f(x)+\frac{n-1}{x_{n}} Q^{\prime} f(x)=0,
$$

The proof is complete.

$$
M_{n} f(x)=M f(x)+\frac{Q^{\prime} f(x)}{x_{n}}=\frac{Q^{\prime} f(x)}{x_{n}} .
$$

Also we shall need the following result.

Lemma 2.7. [5] If $f$ is a twice continuously differentiable function from $\Omega \subset$ $\mathbf{R}_{+}^{n+1}$ into $\mathscr{C} \ell_{0, n}$, and $\overline{B_{h}\left(e_{n}, R_{h}\right)} \subset \Omega$, we obtain

$$
\frac{d}{d R_{h}}\left(\frac{1}{\sinh ^{n} R_{h}} \int_{\partial B_{h}\left(a, R_{h}\right)} f d \sigma_{h}\right)=\frac{1}{\sinh ^{n} R_{h}} \int_{B_{h}\left(a, R_{h}\right)} \Delta_{h} f d x_{h} .
$$

Next we deduce that any eigenfunction of the Laplace-Beltrami operator is a $Q$-part of some hypermonogenic function. The theorem is formulated only for a ball but similar theorem holds also for more general star-shaped domains (cf. [2]).

Theorem 2.8. [2] Let $h: B_{h}(a, R) \rightarrow \mathscr{C} \ell_{0, n-1}$ be a solution of the equation

$$
\Delta_{l b} h(x)=-(n-1) h(x) .
$$

There exists a hypermonogenic function $f: B_{h}(a, R) \rightarrow \mathscr{C} \ell_{0, n}$ satisfying $h=Q f$ on $B_{h}(a, R)$.

Now we may start to give the proof for the Theorem 2.1. First we show that the statement (1) implies (2).

Lemma 2.9. Let $h: \Omega \rightarrow \mathscr{C} \ell_{0, n-1}$ be a solution of

$$
\Delta_{l b} h(x)=-(n-1) h(x)
$$

on $\Omega$ and let $\overline{B_{h}\left(a, R_{h}\right)} \subset \Omega$. Then

$$
h(a)=\frac{1}{(n-1) \omega_{n+1} \sinh R_{h} \int_{0}^{R_{h}} \sinh ^{n-2}(t) d t} \int_{\partial B_{h}\left(a, R_{h}\right)} h(x) d \sigma_{h}(x)
$$

Proof. Let $f$ be a hypermonogenic function satisfying $Q f=h$ on $B_{h}\left(a, R_{h}\right)$. Applying Proposition 2.3 and Theorem 2.4 we obtain

$$
\begin{aligned}
& \omega_{n+1} Q f(a) \\
& =\int_{\partial B_{h}\left(a, R_{h}\right)} Q\left(\frac{\left(\overline{(x-\tau(a, x))} \cosh d_{h}(x, a)-a_{n} \sinh ^{2} d_{h}(x, a) e_{n}\right.}{a_{n} x_{n}^{n} \sinh ^{n+1} d_{h}(x, a)} \nu(x) f(x)\right) d S(x) .
\end{aligned}
$$


Since on the ball $B_{h}\left(a, R_{h}\right)$ the unit normal field is given by $\nu(x)=\frac{x-\tau(a, x)}{R_{e}\left(a, R_{h}\right)}$, we infer

$$
\begin{aligned}
& \omega_{n+1} Q f(a) \\
& =\int_{\partial B_{h}\left(a, R_{h}\right)} Q\left(\frac{R_{e}\left(a, R_{h}\right) \overline{\nu(x)} \cosh d_{h}(x, a)-a_{n} \sinh ^{2} d_{h}(x, a) e_{n}}{a_{n} x_{n}^{n} \sinh ^{n+1} d_{h}(x, a)} \nu(x) f(x)\right) d S(x)
\end{aligned}
$$

Since $\overline{\nu(x)} \nu(x)=1$, we obtain

$$
\begin{aligned}
\omega_{n+1} Q f(a)= & \frac{R_{e}\left(a, R_{h}\right) \cosh R_{h}}{a_{n} \sinh ^{n+1} R_{h}} \int_{\partial B_{h}\left(a, R_{h}\right)} Q f(x) d \sigma_{h} \\
& -\frac{1}{\sinh ^{n-1} R_{h}} \int_{\partial B_{h}\left(a, R_{h}\right)} Q\left(\frac{e_{n} \nu(x) f(x)}{x_{n}^{n}}\right) d S(x) .
\end{aligned}
$$

Since $R_{e}\left(a, R_{h}\right)=a_{n} \sinh R_{h}$, by virtue of Lemma 2.6 we have

$$
\omega_{n+1} Q f(a)=\frac{\cosh R_{h}}{\sinh ^{n} R_{h}} \int_{\partial B_{h}\left(a, R_{h}\right)} Q f(x) d \sigma_{h}-\frac{1}{\sinh ^{n-1} R_{h}} \int_{B_{h}\left(a, R_{h}\right)} Q f(x) d x_{h} .
$$

Using Lemma 2.7 and the assumption we have

$$
\begin{aligned}
\omega_{n+1} Q f(a)= & \frac{\cosh R_{h}}{\sinh ^{n} R_{h}} \int_{\partial B_{h}\left(a, R_{h}\right)} Q f(x) d \sigma_{h} \\
& -\frac{\sinh R_{h}}{n-1} \frac{d}{d R_{h}}\left(\frac{1}{\sinh ^{n} R_{h}} \int_{\partial B_{h}\left(a, R_{h}\right)} Q f(x) d \sigma_{h}\right) .
\end{aligned}
$$

The equation in above give us the differential equation

$$
\sinh \left(R_{h}\right) g^{\prime}\left(R_{h}\right)+(n-1) \cosh \left(R_{h}\right) g\left(R_{h}\right)=C,
$$

where $C=(n-1) Q f(a)$ and

$$
g\left(R_{h}\right)=\frac{1}{\omega_{n+1} \sinh ^{n} R_{h}} \int_{\partial B_{h}\left(a, R_{h}\right)} Q f(x) d \sigma_{h} .
$$

The general solution of this equation is

$$
g\left(R_{h}\right)=\frac{C \int_{0}^{R_{h}} \sinh ^{n-2} t d t+C_{0}}{\sinh ^{n-1}\left(R_{h}\right)} .
$$

Since $g$ is a continuous function, we have

$$
\lim _{R_{h} \rightarrow 0+} g\left(R_{h}\right)=Q f(a)
$$

and then $C_{0}=0$. The proof is complete.

We show next that the statement (2) implies (3).

Lemma 2.10. Assume

$$
h(a)=\frac{1}{\omega_{n+1} \sinh R_{h} \int_{0}^{R_{h}} \sinh ^{n-2}(t) d t} \int_{\partial B_{h}\left(a, R_{h}\right)} h(x) d \sigma_{h}(x) .
$$

Then

where

$$
h(a)=\frac{n-1}{\omega_{n+1} \phi\left(R_{h}\right)} \int_{B_{h}\left(a, R_{h}\right)} Q f(x) d x_{h},
$$

$$
\phi\left(R_{h}\right)=(n-1) \cosh R_{h} \int_{0}^{R_{h}} \sinh ^{n-2}(t) d t-\sinh ^{n-1} R_{h} .
$$


Proof. Using Lemma 2.7 we have

$$
-\frac{n-1}{\sinh ^{n} R_{h}} \int_{B_{h}\left(a, R_{h}\right)} h(x) d x_{h}=\frac{d}{d R_{h}}\left(\frac{1}{\sinh ^{n} R_{h}} \int_{\partial B_{h}\left(a, R_{h}\right)} h(x) d \sigma_{h}\right) .
$$

By the assumptions

$$
\begin{aligned}
& -\frac{n-1}{\omega_{n+1} \sinh ^{n} R_{h}} \int_{B_{h}\left(a, R_{h}\right)} h(x) d x_{h} \\
& =\left(\frac{1}{\sinh R_{h}}-(n-1) \frac{\cosh R_{h} \int_{0}^{R_{h}} \sinh ^{n-2} t d t}{\sinh ^{n} R_{h}}\right) h(a) .
\end{aligned}
$$

Then

$$
h(a)=\frac{n-1}{\omega_{n+1}(n-1) \cosh R_{h} \int_{0}^{R_{h}} \sinh ^{n-2}(t) d t-\sinh ^{n-1} R_{h}} \int_{B_{h}\left(a, R_{h}\right)} h(x) d x_{h},
$$

and the proof is complete.

We show next that (3) implies (2). First we need the following lemma.

Lemma 2.11. Let $T: B_{h}\left(e_{n}, R_{h}\right) \rightarrow B_{h}\left(a, R_{h}\right)$ be the mapping

$$
T(x)=a_{n} x+P a,
$$

where $a \in \mathbf{R}_{+}^{n+1}$. Then $T$ is diffeomorphism, and the following transformation rules hold:

(a) $\int_{\partial B_{h}\left(a, R_{h}\right)} f(y) d \sigma_{h}(y)=\int_{\partial B_{h}\left(e_{n}, R_{h}\right)} f \circ T^{-1}(x) d \sigma_{h}(x)$,

(b) $\int_{\partial B_{h}\left(a, R_{h}\right)} f \circ T(x) d \sigma_{h}(x)=\int_{\partial B_{h}\left(e_{n}, R_{h}\right)} f(y) d \sigma_{h}(y)$,

(c) $\int_{B_{h}\left(a, R_{h}\right)} h(y) d y_{h}=\int_{B_{h}\left(e_{n}, R_{h}\right)} h \circ T^{-1}(x) d x_{h}$,

(d) $\int_{B_{h}\left(a, R_{h}\right)} h \circ T(x) d x_{h}=\int_{B_{h}\left(e_{n}, R_{h}\right)} h(y) d y_{h}$.

That allows us to prove the following lemma.

Lemma 2.12. Assume

$$
h(a)=\frac{n-1}{\omega_{n+1} \phi\left(R_{h}\right)} \int_{B_{h}\left(a, R_{h}\right)} h(x) d x_{h} .
$$

Then

$$
h(a)=\frac{1}{\omega_{n+1} \sinh R_{h} \int_{0}^{R_{h}} \sinh ^{n-2}(t) d t} \int_{\partial B_{h}\left(a, R_{h}\right)} h(x) d \sigma_{h}(x) .
$$

Proof. Using the previous proposition we infer

$$
h(a)=\frac{n-1}{\omega_{n+1} \phi\left(R_{h}\right)} \int_{B_{h}\left(e_{n}, R_{h}\right)} h \circ T^{-1} h(x) d x_{h} .
$$

Using the polar coordinates we have

$$
h(a)=\frac{n-1}{\omega_{n+1} \phi\left(R_{h}\right)} \int_{0}^{R_{h}} \int_{\partial B_{h}\left(e_{n}, t\right)} h \circ T^{-1}(x) d \sigma_{h}(x) d t .
$$

Then

$$
\omega_{n+1} \phi\left(R_{h}\right) h(a)=(n-1) \int_{0}^{R_{h}} \int_{\partial B_{h}\left(e_{n}, t\right)} h \circ T^{-1}(x) d \sigma_{h}(x) d t
$$


Since

$$
\phi^{\prime}\left(R_{h}\right)=(n-1) \sinh R_{h} \int_{0}^{R_{h}} \sinh ^{n-2}(t) d t,
$$

using Lemma 2.7 we have

$$
h(a)=\frac{1}{\omega_{n+1} \sinh R_{h} \int_{0}^{R_{h}} \sinh ^{n-2}(t) d t} \int_{\partial B_{h}\left(e_{n}, R_{h}\right)} h \circ T^{-1}(x) d \sigma_{h}(x) .
$$

Then using the (a)-part of the preceding proposition we have

$$
h(a)=\frac{1}{\omega_{n+1} \sinh R_{h} \int_{0}^{R_{h}} \sinh ^{n-2}(t) d t} \int_{\partial B_{h}\left(a, R_{h}\right)} h(y) d \sigma_{h}(y) .
$$

The proof is complete.

Lastly we deduce that (2) implies (1).

Lemma 2.13. Assume

$$
h(a)=\frac{1}{\omega_{n+1} \sinh R_{h} \int_{0}^{R_{h}} \sinh ^{n-2}(t) d t} \int_{\partial B_{h}\left(a, R_{h}\right)} h(x) d \sigma_{h}(x) .
$$

Then

for $x \in B_{h}\left(a, R_{h}\right)$.

$$
\Delta_{l b} h(x)=-(n-1) h(x)
$$

Proof. Since

$$
\frac{d}{d R_{h}} \frac{\sinh ^{n-1} R_{h}}{\int_{0}^{R_{h}} \sinh ^{n-2}(t) d t}=\frac{\sinh ^{n-2} R_{h} \phi\left(R_{h}\right)}{\left(\int_{0}^{R_{h}} \sinh ^{n-2}(t) d t\right)^{2}},
$$

using Lemma 2.7 we obtain

$$
\begin{aligned}
0= & \frac{\sinh ^{n-2} R_{h} \phi\left(R_{h}\right)}{\left(\int_{0}^{R_{h}} \sinh ^{n-2}(t) d t\right)^{2}} \frac{1}{\sinh ^{n} R_{h}} \int_{\partial B_{h}\left(a, R_{h}\right)} h(x) d \sigma_{h}(x) \\
& +\frac{\sinh ^{n-1} R_{h}}{\int_{0}^{R_{h}} \sinh ^{n-2}(t) d t} \frac{1}{\sinh ^{n} R_{h}} \int_{B_{h}\left(a, R_{h}\right)} \Delta_{l b} h(x) d x_{h} .
\end{aligned}
$$

Since (2) and (3) are equivalent, we obtain the formula

$$
\int_{\partial B_{h}\left(a, R_{h}\right)} h(x) d \sigma_{h}(x)=(n-1) \frac{\sinh R_{h} \int_{0}^{R_{h}} \sinh ^{n-2}(t) d t}{\phi\left(R_{h}\right)} \int_{B_{h}\left(a, R_{h}\right)} h(x) d x_{h} .
$$

Then

$$
\begin{aligned}
0= & \frac{(n-1) \phi\left(R_{h}\right)}{\left(\int_{0}^{R_{h}} \sinh ^{n-2}(t) d t\right)^{2}} \frac{1}{\sinh ^{2} R_{h}} \frac{\sinh R_{h} \int_{0}^{R_{h}} \sinh ^{n-2}(t) d t}{\phi\left(R_{h}\right)} \int_{B_{h}\left(a, R_{h}\right)} h(x) d x_{h} \\
& +\frac{1}{\int_{0}^{R_{h}} \sinh ^{n-2}(t) d t} \frac{1}{\sinh R_{h}} \int_{B_{h}\left(a, R_{h}\right)} \Delta_{l b} h(x) d x_{h},
\end{aligned}
$$

that is,

$$
\frac{1}{\sinh R_{h} \int_{0}^{R_{h}} \sinh ^{n-2}(t) d t} \int_{B_{h}\left(a, R_{h}\right)}\left(\Delta_{l b} h(x)+(n-1) h(x)\right) d x_{h}=0 .
$$


Since $R_{h}$ is arbitrary, we obtain that

$$
\Delta_{l b} h(a)+(n-1) h(a)=0 .
$$

The proof is complete.

\section{References}

[1] ERIKsson, S.-L.: Integral formulas for hypermonogenic functions. - Bull. Belg. Math. Soc. Simon Stevin 11, 2004, 705-717.

[2] Eriksson, S.-L., and H. Leutwiler: Hypermonogenic functions. - In: Clifford Algebras and their Applications in Mathematical Physics 2, Birkhäuser, Boston, 2000, 287-302.

[3] Eriksson, S.-L., and H. Leutwiler: On hyperbolic function theory. - Adv. Appl. Clifford Algebras 18:3-4, 2008, 587-598.

[4] ERIKSSON, S.-L., and H. Leutwiler: Introduction to hyperbolic function theory. - In: Clifford Algebras and Inverse Problems (Tampere 2008), Tampere Univ. of Tech. Institute of Math. Research Report No. 90, 2009, 1-28.

[5] Eriksson, S.-L, and H. Leutwiler: Hyperbolic harmonic functions and their function theory. - In: Potential Theory and Stochastics in Albac, 2009, 85-100.

[6] Eriksson, S.-L., and H. Orelma: A mean-value theorem for hyperbolic harmonic functions. - Submitted.

[7] Leutwiler, H.: Appendix: Lecture notes of the course "Hyperbolic harmonic functions and their function theory". - In: Clifford algebras and potential theory, Univ. Joensuu Dept. Math. Rep. Ser. 7, Univ. Joensuu, Joensuu, 2004, 85-109.

[8] Leutwiler, H.: Quaternionic analysis in $\mathbf{R}^{3}$ versus its hyperbolic modification. - In: Clifford Analysis and its Applications, edited by F. Brackx et al., Kluwer, Dordrecht 2001, 193-211.

Received 26 November 2009 